\title{
INNOVATION ET CONVENTIONS SOCIALES \\ USAGES DU NUMÉRIQUE ET CAPITALISME FINANCIER
}

Dominique Boullier*

Les innovations ont vocation à remettre en cause les conventions sociales, pense-t-on habituellement. Ce n'est pas faux à la condition de préciser que les innovations ne réussissent que dans la mesure où elles produisent et s'appuient elles-mêmes sur des conventions, c'est -à-dire des situations stabilisées pour étendre le marché et permettre l'appropriation par les consommateurs. Le nouvel esprit du capitalisme dans lequel nous sommes, celui du capitalisme financier, constitue de ce point de vue une rupture puisqu'il valorise avant tout la fluidité du capital et des biens et même des savoirs-faire, qui doivent sans cesse être remis en cause sous forme de flexibilité généralisée des travailleurs comme des consommateurs. Or, nous voulons montrer ici que cette tendance générale imposée par le capitalisme financier risque de tuer toute possibilité de constituer des conventions sociales, de ne plus permettre ainsi la constitution d'apprentissages durables et de ce fait de mettre en péril toute extension possible du marché lui-même. La technologie du numérique est particulièrement adaptée à cette tendance lourde à la fluidité car elle est adaptable en permanence et la vitesse de diffusion des nouvelles versions de logiciel finit par rendre très

* Antropólogo, professor em Ciência da Informação e da Comunicação na Universidade de Tecnologia de Compiègne (França). Diretor da unidade de pesquisas Costech (Connaissances , Organisation , Systèmes techniques) e responsável pedagógico do DESS à distância Dicit. (dominique.boullier@ utc.fr) 
difficile le maintien à niveau de larges groupes de population. On prétend lutter ainsi contre la fracture numérique alors que l'on fait entrer des populations entières dans le cercle infernal de l'instabilité des innovations, elles-mêmes non justifiées du point de vue des usages.

\section{LA THÉORIE DES CONVENTIONS}

La tradition économique a tenté de traiter l'innovation dans le cadre de la théorie standard à l'aide des concepts qui la constituent :une théorie de l'agent rationnel et calculateur d'un côté et de l'autre, et de façon séparée, une théorie de la coordination des agents limitée à l'équilibre général constituant le marché. La théorie des conventions a contesté à la fois la séparation entre ces deux approches et la disparition de toute dimension normative dans la coordination des acteurs aussi bien que dans leurs critères de décision. Lessig par ailleurs, partant d'une approche juridique et politique, a montré comment la régulation se fait toujours à la fois en termes de marché, en termes de normes, en termes de règles ou en termes de technique : toute coordination durable entre acteur repose sur un équilibre entre ces dimensions, et tout changement dans l'un des facteurs (notamment à travers les technologies, et en particulier internet) oblige à une recomposition des arbitrages avec les autres formes de régulation. L'innovation suscite aussitôt toute cette réorganisation que la théorie des conventions permet de formaliser. On parle alors non pas de " marché " mais de composition de marchés, toujours pluriels. Les marchés sont des épreuves organisées pour qualifier les biens non seulement sur le plan des prix mais aussi sur les autres dimensions, dont les dimensions normatives. Les conventions de qualité étudiées par Eymard Duvernay, constituent une forme de coordination qui incorporent une grande quantité de valeurs d'ordre différent et qui reposent sur des dispositifs techniques ou réglementaires pour mettre à l'épreuve ces qualités. Les tests d'usage et les tests ergonomiques que nous conduisons dans notre laboratoire parisien (Lutin) relèvent de cet effort pour rendre conventionnels d'autres qualités de biens. Toutes ces conventions nécessitent ainsi, avant même la mise sur le marché, des investissements de forme (Thévenot), c'est à dire de créer et de faire reconnaitre, des formats de qualification qui sont par exemple 
en jeu dans toutes les normes ISO ou dans les labels pour les produits alimentaires : cette qualification demande un lourd travail de mesures, d'objectivation, d'institution de partenariats et de chaines de production de ces qualités, de travail de l'opinion pour faire partager les nouvelles normes, etc.). On le voit, une innovation qui interviendrait dans un désert, sans tenir des règles de sécurité, des techniques de merchandising, des exigences légales diverses, des performances techniques objectivées par des instituts indépendants (ex : les décibels d'un réfrigérateur), des réseaux de distribution, etc.. n'aurait à peu près aucune chance de survivre. Parmi ces conditions et ces conventions, le niveau de connaissance de l'utilisateur peut tout changer : saura-t-il l'utiliser spontanément (mais quel background, quelle expérience cela suppose-t-il quand même et pour quelles populations), faudra-t-il le former, devra-t-on lui fournir une aide imprimée, une aide en ligne, une hot-line, un service après- vente et quels seront les efforts à répartir entre tous ces systèmes d'aide. Autant de questions qui supposent ainsi de s'affronter au degré de stabilisation de conventions d'usage parmi les utilisateurs voire de créer ces conventions.

\section{LES TYPES D'INNOVATION}

Il est d'usage de différencier les innovations incrémentales et les innovations de rupture pour penser les stratégies qui les accompagnent. Une innovation incrémentale s'appuiera sur les conventions existantes pour un produit donné et ne cherchera pas à les remettre en cause mais seulement à apporter une variation à la marge. Cette démarche permet ainsi d'approfondir le marché en le faisant durer plus longtemps, ou en trouvant de nouveaux clients qui sont intéressés par les options parfois très élémentaires qui sont apportées. Nous avons ainsi participé à la redéfinition des couleurs d'un poste téléphonique, qui grâce à son look fluo a permis de conquérir toute une clientèle jeune alors que la technologie elle-même n'avait rien d'innovante et ne changeait pas par rapport au produit précédent. Ce cas est une situation limite que beaucoup refuseront d'appeler innovation, sous prétexte que la composante technologique y est faible : c'est un erreur d'autant plus dans les marchés contemporains sous domination du capitalisme financier, comme nous le verrons plus loin, 
largement déterminés par le look and feel, typique d'un monde de l'opinion ou les impressions immédiates sont décisives pour faire la décision.

L'innovation de rupture quand à elle, prétend conquérir les marchés précisément en se démarquant des traditions et des conventions au point de rendre tout calcul d'avantage comparatif impossible. Les études de diffusion de l'innovation (Rogers) ont montré à quel point il était nécessaire de s'appuyer sur quelques leaders d'opinion, les " early adopters ", pour qui, par principe, toute innovation doit être essayée. Cette tendance faiblement présente malgré tout dans une population donnée, tend à être renforcée par les médias qui vont avoir tendance à valoriser a priori tous ces adopteurs précoces présentés comme les modèles à suivre. Là encore, des dispositifs qui construisent l'opinion peuvent aider à constituer une rupture comme une norme sociale désirable contre les autres conventions établies. Mais il faut souligner à quel point les véritables innovations de rupture sont rares car il est extrêmement difficile de remettre en cause des conventions établies pendant des dizaines, ancrées dans les représentations mais aussi et surtout dans des dispositifs techniques, des infrastructures, des circuits d'intermédiaires et de professionnels, des modèles économiques, etc. L'exemple classique du clavier Dvorak, inventé en 1932, est souvent rappelé pour le démontrer. Considéré comme ergonomiquement plus pertinent et efficace puisque que le clavier actuel a été inventé pour ralentir la frappe mécanique à partir de 1870 , le clavier Dvorak n'a jamais percé car ce ne sont jamais les qualités intrinsèques du produit qui font son succès mais toujours ses qualités extrinsèques (Akrich, Callon, Latour), c'est à dire celles du réseau d'alliés qu'il a constitué. Le clavier ordinaire possède avec lui des alliés aussi puissants que des armées de secrétaires formées à la dactylo ou des constructeurs de machines. Changer ces conventions est nettement plus difficile que de produire de l'innovation " techniquement " plus performante. Les changements de convention portent notamment sur les apprentissages nécessaires pour les clients ou pour le public en général et tout cela est particulièrement lourd à mobiliser et à transformer. Les investissements cognitifs effectués par des utilisateurs ordinaires doivent être durables et conduire à produire des routines de façon alléger la charge mentale. Toute remise en cause doit alors être particulièrement justifiée et 
ne peut être renouvelée à tout moment : la stabilisation de ces conventions que nous dirons socio-cognitives est une condition pour que le marché puisse s'appuyer sur une base quasi naturelle, celle que l'on appelle parfois dans les études marketing, la base équipée , pour parler du matériel, et qu’il faudrait étendre ici à tout l'équipement cognitif.

\section{CONVENTIONS ET INNOVATION DANS LE CAPITALISME INDUSTRIEL}

Le troisième âge du capitalisme, au-delà de sa phase d'accumulation primitive, puis du capitalisme marchand, puis industriel apparaît comme une remise en cause radicale des stratégies d'innovation, voire, pour ceux qui pensent en termes d'économie industrielle, comme une vraie menace pour toute stratégie industrielle.

Nous prendrons délibérément un exemple dans le monde de la consommation grand public, sans ignorer que les processus peuvent être assez différents dans l'univers des biens d'équipements industriels par exemple. Le cas de Eastman est exemplaire parce que précisément son indéniable innovation technique (l'appareil photo comportant une pellicule tendue dans un boîtier portable et enduite d'une couche argentique) n'a pu être conçue qu'en fonction d'une autre innovation radicale, celle du photographe amateur. C'est bien le client (nous dirions le marché mais ce serait trop général) photographe amateur qui a été inventé et qui a guidé tous les choix techniques de Eastman. A tel point que toute la chaîne de distribution a été inventée en même temps (la remise des pellicules à un distributeur et le retour des tirages au client)et que ce dispositif technicocommercial a survécu jusqu'à nos jours. Pendant cent ans, une convention sociale durable s'est installée qui permettait à de nouveaux clients d'être pris en charge aisément et d'avoir confiance dans la qualité du processus. La révolution numérique est semble-t-il en train de remettre en cause ce modèle, mais ce n'est pas certain que la prise en charge habituelle ne puisse pas survivre après les premières phases euphoriques des « early adopters " pour le développement des photos avec son imprimante personnelle.

Dans le même ordre d'idée, il faut noter à quel point le développement du marché automobile a été facilité par un travail considérable de mises en 
forme de conventions (d'investissements de forme) particulièrement stables, à l'échelle internationale, entre concurrents et états différents. On ne peut pas comprendre l'extension du marché automobile sans tenir compte du développement massif d'infrastructures, sans les chaînes de production sécurisée de carburants, (parfois au prix de guerre et de monopoles bien gardés ou d'ententes bien organisées, bien loin des supposées lois du marché), sans la production de règles telles que le code de la route, appliquées par des matériels de signalisation, des lois, des policiers, etc.., et surtout enseignés par des écoles de conduite. Les constructeurs automobiles ont réussi cette prouesse de faire payer les utilisateurs de leur produit pour se former à l'utilisation du produit (car on y apprend aussi à conduire !mais avec le contrôle de l'état). Cet investissement lourd pour chaque conducteur ne pouvait être accepté qu'à la condition qu'une stabilité était garantie. De fait, la voiture a finalement peu changé depuis cent ans, malgré tous les ajouts d'option et d'amélioration : elle est toujours faite principalement pour 4 passagers même si dans les villes, on y roule le plus souvent seul, elle consomme toujours du pétrole alors qu'on sait qu'il va s'épuiser rapidement, les pédales sont restées les mêmes et les vitesses ont fini d'être normalisées sur le plan international durant les années 60 . les industriels se sont ainsi entendus pour éviter toute innovation radicale qui n'aurait pas profité des investissements de forme effectués dans les infrastructures, dans la réglementation et dans le corps des conducteurs qui, 50 ans après leur permis de conduire, peuvent toujours utiliser un système très voisin.

\section{LES CONVENTIONS DANS LE CAPITALISME FINANCIER}

Les conventions sont en elles-mêmes déstabilisées par la nature même du marché financier. Le marché financier est un marché particulier, de même que le marché du travail, par rapport aux autres marchés et les conventions qui le régissent sont particulières même si elles ont précisément tendance à s'imposer à tous les autres marchés désormais.

La qualité essentielle des biens sur le marché financier est devenue leur fluidité. Tout bien financier doit pouvoir être vendu instantanément et racheté quelques instants plus tard, les réseaux informatiques offrant 
toutes les conditions techniques pour réaliser ces conditions maximales de fluidité. Les technologies d'information sont ainsi au cœur de ce nouveau mode de production qu'est le capitalisme financier. De ce point de vue, toute immobilisation fait perdre de la valeur et les stratégies de dématérialisation de l'économie trouvent aussi leur source dans ce principe dominant. La coordination des acteurs ne se fait plus sur la valeur des biens matériels, des investissements divers, du type des moyens de production, qui par définition deviennent négatifs puisqu'ils immobilisent du capital : il faut d'ailleurs rémunérer le capital pour cette perte de fluidité, ce qui explique les exigences de rentabilité invraisemblables fixées désormais aux entreprises. Il est en effet toujours plus avantageux pour un acteur financier de placer son capital dans des biens vendables immédiatement, dans des valeurs financières, sauf si les anticipations sur les entreprises permettent de penser que la revente sera possible avec une marge suffisante. Or, ces anticipations ne reposent sur rien d'autre que sur la perception des actions possibles des autres acteurs du marché financier. Cette perception est ellemême organisée par les médias, par les réputations qui circulent et qui constituent ce que André Orléan appelle l'économie d'opinion. On peut ainsi plus facilement comprendre que les bulles ne sont pas échecs des marchés financiers à se réguler mais bien plutôt leur règle normale de base. La bulle internet a précédé une future bulle biotechnologique mais pendant ce temps, le marché de l'immobilier lui-même accaparé par les investisseurs que sont les fonds de pension est soumis à la même loi. Les économistes ont bien raison de s'intéresser au statut des croyances dans la construction des marchés car le marché financier est un producteur massif de croyances, impossibles à fonder dans d'autres critères que les perceptions réciproques.

\section{Une méta-convention: la fluidité permanente, la cité par projets}

Ce modèle propre aux marchés financiers finit par façonner toute l'économie depuis que la puissance des échanges financiers a été libérée en 1971 par la fin des accords de Brenton Woods et de l'étalon or pour le dollar. Tout d'abord sous la forme d'une méta-convention, pourrait 
on dire, celle qui consiste à survaloriser la fluidité et par conséquent la déstabilisation constante de toute convention qui constitue en elle-même une immobilisation trop coûteuse pour le capital. Ce modèle dépasse de loin la sphère des échanges financiers pour régir les relations entre entreprises, avec les relations imposées par exemple aux sous traitants, avec la flexibilité des localisations (on rompt les liens trop durables avec les territoires), mais aussi dans la relation avec les salariés dont on organiser la précarité. En France, une catégorie particulière incarne bien ce nouveau, les intermittents du spectacle, rémunérés seulement quelques mois dans l'année spectacle par spectacle et toujours à statut précaire... durable. Or, une grande partie de ces personnels revendiquent aussi ce statut précaire, au nom de leur liberté, contre le salariat aliénant, au profit d'une vision du travail organisé par des projets. Ce monde dominé par l'idéologie du projet a été décrit par Boltanski et Chiapello qui en font le cœur même de leur " nouvel esprit du capitalisme ". Le capitalisme a ainsi intégré les critiques antiautoritaires et antitraditionnelles qui lui ont été faites et a proposé, notamment à toutes les professions à forte composante intellectuelle, d'organiser elles -mêmes leur travail selon des projets, sans cess reconfigurables, par définition instables. Ce modèle correspond ainsi aux valeurs mêmes de ces personnels, paraît conforme à leur statut jusqu'au moment où ils commencent à mesurer qu'ils s'exploitent eux-mêmes, en fournissant une masse de travail non rémunéré, qu'ils courent après les projets, après les re-engineering constants, en se trouvant totalement isolés et sans aucune maitrise sur le processus. Ce modèle pénètre ainsi les esprits en profondeur et fait admettre la nécessité de se remettre en cause à un rythme effréné sans garantie pour l'avenir.

On en trouve la trace dans les discours sur ce que nous avons appelé dès 1983, "la tyrannie du retard ". Tous les pays se trouvent soumis à des politiques de rattrapage de retard par rapport à d'autres considérés comme en avance, sans autre réflexion stratégique que celle qui consiste à promouvoir la consommation d'appareils électroniques, d'ordinateurs ou d'Internet. Dans ces conditions, c'est un mimétisme général qui s’installe. La socialisation des nouveaux publics aux nouvelles technologies se fait dans l'urgence, en leur faisant apprendre des techniques, des logiciels totalement inadaptés à leurs contextes d'usage et à leurs préoccupations, 
en les mettant ainsi très vite en situation de peur de l'échec qui devient équivalent à un déclassement social.

\section{Les biens immatériels}

La deuxième conséquence de cette domination du capitalisme financier porte sur la nature des biens qui deviennent susceptibles de valeur, c'est à dire ceux qui peuvent être vendus aisément. On trouve dans cette catégorie tous les biens dits immatériels. "L'économie immatérielle " ou encore celle que l'on dit du " capitalisme cognitif " n'est en fait rien d'autre que celle du capitalisme financier imposant ses exigences de fluidité et de flexibilité et choisissant pour cela des biens spécifiques. Ainsi les brevets et les marques sont les deux ressources importantes de valorisation d'une entreprise. Par définition, ils sont difficiles à apprécier et c'est alors tout le travail des comptables de donner consistance à cette valeur évanescente, en les qualifiant de "goodwills ». L'arbitraire le plus total ou plutôt la dépendance extrême à l'opinion (à travers les réputations), de ces évaluations a conduit à des effondrements spectaculaires comme ce fut le cas de Vivendi Universal. La valeur intrinsèque d'une marque comme d'un brevet n'a pas de sens, ce ne sont que les anticipations sur la valeur de revente qui constituent la valeur.

D'autres biens sont cependant intéressants pour le capitalisme financier pour leur fluidité : les services ou les fichiers clients par exemple. On ne fait plus, on fait faire, malgré les éventuels coûts de transaction définis par Williamson, mais ici fortement relativisés par les coûts à la revente des actifs financiers dans ces entreprises, devenu le critère essentiel. Les fichiers clients constituent en revanche une valeur potentielle énorme, ce qui a conduit des entreprises lors de la bulle internet à valoriser ces fichiers alors même qu'ils ne vendaient rien ou à perte. On peut en effet valoriser le possible accès à des données personnelles et à des profils qui permettront de cibler des offres très variées. Plus généralement, les médias et les réseaux constituent des biens recherchés dans cette économie financiarisée car c'est à travers eux que s'échangent et se construisent les réputations. 


\section{EFFETS SUR L'INNOVATION}

Dans un tel contexte, on conçoit aisément que les innovation radicales qui supposeraient des investissements à long terme dans la recherche ne soient pas du tout privilégiés. Ce sont au contraire les innovations incrémentales, qui changent avant l'image des produits ou quelques aspects des services sur une base technique identique ou seulement enrichie qui sont essentiels. La technologie et ses innovations ne produisent plus en elles-mêmes la valeur : lorsque les compagnies de téléphonie mobile offrent l'appareil contre un abonnement, on mesure à quel point l'économie s'est détachée des biens matériels.

On pourrait donc considérer que cette incapacité à financer des innovations radicales constitue un gage de stabilité pour les conventions d'usage, et permettent aux utilisateurs d'améliorer doucement les performances de dispositifs qu'ils ont appris à utiliser pendant plusieurs années. Or, il n'en est rien car la tendance à valoriser l'innovation incrémentale se situe du côté de l'instabilité permanente. Il faut au contraire organiser une perpétuelle remise en cause des façons de faire, sur la même plate-forme technologique apparemment, pour vendre à nouveau des versions successives d'un même produit, alors même que pour la plupart des utilisateurs de base, les versions précédentes étaient déjà largement satisfaisantes ou même trop puissantes. Le principe de ce type d'innovation repose sur des événements (les lancements de versions de logiciels par exemple), sur une mise sous tension de tous les consommateurs, qui finissent par se convaincre qu'ils ne peuvent plus se passer de ces nouvelles versions.

Le coût cognitif de ces changements permanents est très important. De nombreux nouveaux clients , pleins de bonne volonté, sont vides découragés par l'exigence constante dans l'informatique par exemple d'ajouter de nouveaux éléments, de changer de versions, de traiter des problèmes d'incompatibilité ainsi créés, etc. Toute cette pression constitue selon nous au moins $50 \%$ de la fracture numérique que l'on prétend combattre en diffusant les usages de ces technologies de l'information. En effet, le coût du désapprentissage est énorme. Les nouveaux savoir-faire ne s'accumulent pas à côté des précédents : ils les remettent en cause et nécessitent un 
travail d'acquisition, de révolution personnelle, pour accepter de perdre ce qui avait constitué pendant des années, une évidence, un état naturel. On pourra faire le parallèle avec la procédure de désinstallation qui parfois se déroule mal et rend impossible l'installation d'un nouveau matériel ou système. Ce travail est de plus en plus difficile avec l'âge, y compris pour des raisons de calcul quant à l'intérêt d'un tel travail lorsqu'on se sent proche de la fin de sa vie. Mais il est encore plus certain que lorsque cet effort a été effectué une fois, l'obligation de faire à nouveau ce désapprentissage tous les cinq ans, est insupportable.

De ce fait, ce qui se diffuse avant tout, comme convention susceptible d'être partagée, c'est l'exigence de formation permanente, continue, tout au long de la vie, etc.. Mais ce discours et ces mesures d'aide à la formation auraient de réels effets si les référentiels par rapport auxquels se fait la formation étaient stables. Or, personne ne peut dire à quoi il convient de se former pour être opérationnel et gagnant sur le marché du travail ou tout simplement utilisateur averti dans une échéance de 5 ans !! Les formations demandées sont ainsi de plus en plus brèves et consistent plutôt à s'adapter en quelques heures à un nouveau logiciel par exemple sans prendre le risque d'investir dans des savoirs plus durables. Le paradoxe de l'éducation qui doit apprendre à partir du passé pour s'orienter dans l'avenir était déjà connu. Il devient désormais particulièrement difficile à vivre lorsque ceux qui sont censés orienter l'avenir, les adultes, affirment qu'il convient avant tout de se former à l'incertitude.

Les conventions durables de socialisation aux technologies numériques n'ont pas été construites, c'est certain. Le rattrapage par des formations tous azimuts ou des campagnes de diffusion ne peut s'y substituer. Ce sont en fait les principes même de l'innovation dans le capitalisme financier qui remettent en cause toute valeur à ces formations. On peut dire ainsi que le capitalisme financier détruit les conditions d'une construction durable de conventions permettant la stabilisation puis l'approfondissement des marchés dans le domaine des technologies d'information. Le marché de l'informatique et d'internet ne peut ainsi franchir un certain seuil. C'est ce qui se passe avec l'investissement massif des consommateurs en faveur des portables (ou pour une certaine génération vers les consoles de jeux vidéo). 
Même si ces appareils ont tendance à devenir aussi puissants que certains ordinateurs d'il y a quelques années, ils ont l'avantage de s'appuyer sur une convention d'usage, la téléphonie qui associe plusieurs façons de faire ainsi que des motivations à faire (une réelle pratique déjà ancrée qui s'enrichit et se développe). Dans ce cas, c'est une répartition des marchés entre deux types d'appareils qui est permise. Mais pour les mêmes utilisateurs ordinaires de portables, la situation de "déconnexion " des pratiques est encore en train de se reproduire car on incorpore tant de fonctions au téléphone que ses fonctions de base deviennent difficilement réalisables.

\section{CONCLUSION. DES PISTES AU-DELÀ}

Les seuls remèdes durables pour sortir de cette pression à l'innovation de surface qui perturbe la construction de conventions, sont plutôt à chercher du côté du logiciel libre s’il intègre lui-même cette exigence, cette valeur, sans se laisser happer par une supposée nécessité de versions nouvelles constantes. On peut aussi considérer que les initiatives prises par les organismes publics en matière de diffusion des usages des techniques d'information devraient se traduire par des contraintes plus fortes sur les constructeurs de hard et de soft pour les obliger à s'engager à une stabilité dans les produits. Paradoxalement, les contrats de matériels ou de logiciels ne seraient pas valorisés pour leurs capacités à fournir toujours de nouvelles versions mais au contraire pour leur engagement à stabiliser pendant une durée minimum leurs versions. !

\section{REFERÊNCIAS}

AKRICH, M., CALLON, M., LATOUR, B., A quoi tient le succès des innovations ? L'art de l'intéressement, Gérer et comprendre, Annales des Mines, 11 (1988).

BOLTANSKI , L. et CHIAPELLO E., Le nouvel esprit du capitalisme, Gallimard (NRF), Paris, 1999.

BOLTANSKI, L. et THÉVENOT L., De la justification. Les économies de la grandeur, Gallimard (NRF), Paris, 1991.

BOULLIER, D., Du bon usage d'une critique du modèle diffusionniste. 
Discussion-prétexte des concepts de E.M. Rogers, Réseaux 36 (1989).

BOULLIER, D., et Charlier C., A chacun son Internet. Enquête sur des usagers ordinaires, Réseaux, 86 (1997) 159-181.

BOULLIER, D., Internet et télévision: une convergence tentante et improbable, Les dossiers de l'Audiovisuel 89 (2000) 49-52.

BOULLIER, D., L'urbanité numérique. Essai sur la troisième ville en 2100, L'Harmattan, Paris, 1999.

BOULLIER, Dominique.- "Les conventions pour une appropriation durable des TIC. Utiliser un ordinateur et conduire une voiture ", Sociologie du Travail, 3/2001, p. 369-387.

CALLON, M., Variété et irréversibilité dans les réseaux de conception et d'adoption des techniques, in : Foray, D., Freeman C. (eds), Technologies et Richesse des Nations, Economica, Paris, 1992.

CALLON, M (ed) (1998) The Laws of the Markets, Oxford : Basil Blackwell

LESSIG, Lawrence.- Code and Other Laws in Cyberspace, New-York, Basic Books, 1999.

ORLÉAN, A. (dir.), Analyse économique des conventions, PUF, Paris, 1994.

ORLÉAN, A. Le pouvoir de la finance, 1999, Paris, Ed. Odile Jacob ROGERS, E. M. , Diffusion of Innovations, Free Press, New-York, 1983 (1ère édition : 1963).

EYMARD-DUVERNAY, Favereau O., ORLÉAN, A. SALAIS, R et THÉVENOT, L."L'économie des conventions ou le temps de la reunification dans les sciences sociales ", problèmes économiques, $\mathrm{n}^{\circ}$ 2838, Janvier 2004, La Documentation française, Paris.

THÉVENOT, L., Les investissements de forme, in : THÉVENOT L. (ed) Conventions économiques, CEE-PUF, Paris, 1986, p. 21-71. 
